\title{
Challenges for Service Robots-Requirements of Elderly Adults with Cognitive Impairments
}

\begin{abstract}
Agnieszka Korchut ${ }^{*}$, Sebastian Szklener ${ }^{1}$, Carla Abdelnour ${ }^{2}$, Natalia Tantinya ${ }^{2}$, Joan Hernández-Farigola ${ }^{2}$, Joan Carles Ribes' ${ }^{2}$ Urszula Skrobas', Katarzyna GrabowskaAleksandrowicz', Dorota Szczęśniak-Stańczyk ${ }^{3}$ and Konrad Rejdak ${ }^{1,4}$
\end{abstract}

${ }^{1}$ Department of Neurology, Medical University of Lublin, Lublin, Poland, ${ }^{2}$ Alzheimer Research Center and Memory Clinic of Fundacio ACE, Institut Catala de Neurociencies Aplicades, Barcelona, Catalonia, Spain, ${ }^{3}$ Department of Cardiology, Medical University of Lublin, Lublin, Poland, ${ }^{4}$ Medical Research Center Polish Academy of Sciences, Warsaw, Poland

Objective: We focused on identifying the requirements and needs of people suffering from Alzheimer disease and early dementia stages with relation to robotic assistants.

Methods: Based on focus groups performed in two centers (Poland and Spain), we created surveys for medical staff, patients, and caregivers, including: functional requirements; human-robot interaction, the design of the robotic assistant and user acceptance

OPEN ACCESS

Edited by: Irving E. Vega,

Michigan State University,

United States

Reviewed by:

Francesco Becchi,

Telerobot labs s.r.l., Italy Constança Paúl, University of Porto, Portugal

${ }^{*}$ Correspondence: Agnieszka Korchut agnieszka.korchut@umlub.pl

Specialty section: This article was submitted to Neurodegeneration, a section of the journal Frontiers in Neurology

Received: 31 January 2017 Accepted: 11 May 2017 Published: 01 June 2017

Citation: Korchut A, Szklener S, Abdelnour C, Tantinya N, Hernández-Farigola J, Ribes JC, Skrobas U, GrabowskaAleksandrowicz K, SzczęśniakStańczyk D and Rejdak K (2017)

Challenges for Service Robots-Requirements of Elderly Adults with Cognitive Impairments. Front. Neurol. 8:228. doi: 10.3389/fneur.2017.00228 aspects. Using Likert scale and analysis made on the basis of the frequency of survey responses, we identified users' needs as high, medium, and low priority.

Results: We gathered 264 completed surveys (100 from medical staff, 81 from caregivers, and 83 from potential users). Most of the respondents, almost at the same level in each of the three groups, accept robotic assistants and their support in everyday life. High level priority functional requirements were related to reacting in emergency situations (calling for help, detecting/removing obstacles) and to reminding about medication intake, about boiling water, turning off the gas and lights (almost $60 \%$ of answers). With reference to human-robot interaction, high priority was given to voice operated system and the capability of robotic assistants to reply to simple questions.

Conclusion: Our results help in achieving better understanding of the needs of patients with cognitive impairments during home tasks in everyday life. This way of conducting the research, with considerations for the interests of three stakeholder groups in two autonomic centers with proven experience regarding the needs of our patient groups, highlights the importance of obtained results.

Keywords: service robots, mild cognitive impairment, Alzheimer's disease, user requirements, robotic assistant

\section{INTRODUCTION}

According to the World Health Organization (1), dementia is one of the major causes of disability and dependency among older people. ${ }^{1}$ Altering the way an older person moves around the house, manipulates objects, and obtains sensory perception of the surrounding home environment exerts negative effects on their capacity to execute daily home activities on their own. Such effects are magnified in the case of persons with cognitive impairment.

\footnotetext{
${ }^{1}$ http://www.who.int/mediacentre/factsheets/fs362/en/.
} 
A person with amnestic mild cognitive impairment (MCI) and early stages of Alzheimer disease (AD) has difficulties in instrumental activities of daily living, which depend on memory and executive functioning (2-4).

With the progress of the disease, the help needed for the execution of daily tasks normally increases, leading to a burden on the shoulders of informal caregivers, and in many cases to institutionalization (5).

The number of elderly adults and the incidence of cognitive impairment among them are increasing with the proportion of people aged over 60 being expected to double between 2,000 and 2,050 (6).

As a result, the resources allocated to assisting elderly people will not prove sufficient in the foreseeable future. Robotic assistants could be a way to help people remain healthy and safe in their own homes, ensuring their independence in everyday life. In this context, several social robots, which are human or pet-like robots such as NAO, Paro, KASPAR, PaPeRo, AIBO, and iCat aim at providing social support, engagement, and independence for people with special needs (7-11). Thus, people with cognitive impairment constitute a group, which may particularly benefit from healthcare robots. Currently, the achievements of social robots to date is being projected to achieve psychological and physiological improvement of cognitive impairment conditions among older people and others $(8,12)$. It also enables older people to become more independent (13-15). The 5-year-long observational study of interaction between robots and 139 people with dementia suggests that robots can improve the quality of care for people suffering from dementia (16). Additionally, the demand for social robots is expected to improve the capacity of caregivers in performing daily activities (17-19). A social robot can significantly improve the quality of social services by assisting caregivers (20-24). Some studies identify key areas of needs to be met for persons with dementia (25-27), but studies investigating the needs in relation to robot usage in supporting older adults with cognitive impairment are sparse.

The main objective of this study was to identify user needs and try to classify these findings, which may be a forward step toward changes in robotics for senior adult assistance in cases of amnestic MCI and at early stages of progressive dementia. Carefully assessing needs and matching these to the provided technology can result in higher acceptance rates (28). The study was carried out within the framework of RAMCIP project (full name: robotic assistance for MCI patients) founded by European Programme Horizon 2020. The aim of the project is to create a robot that might support older adults with MCI living independently at home.

\section{MATERIALS AND METHODS}

This study was carried out in accordance with the recommendations of the Ethics Committee of Medical University of Lublin and the Fundació ACE Research Ethics Committee and also under supervision of Ethics Advisory Board established for RAMCIP project needs. Each participant of the focus groups signed written informed consent form in accordance with the Declaration of Helsinki.
Participation in the survey was completely voluntary and informed consent was implied. The survey questionnaires were fulfilled anonymously. All data and results from the questionnaires were presented maintaining participants' individual privacy.

\section{Focus Groups}

At the beginning, we held focus groups in two centers in Poland and Spain.

The workshop with medical staff, conducted in Poland in March 2015, was attended by seven medical doctors and one nurse from a Neurology Department with experience among patients suffering from cognitive impairment.

At the same time, a workshop for caregivers was conducted in Spain at the Diagnostic Unit of the Fundació ACE, Barcelona Alzheimer Treatment and Research Center; with 10 participants.

Focus groups were conducted by a moderator who followed the prepared plan. Participants exchanged their ideas based on their experience and knowledge.

\section{Surveys}

The data collected during focus groups were used to create the survey questionnaire for medical staff, potential users, and their caregivers.

The answers from surveys were sourced from two autonomic centers: Medical University of Lublin (LUM) and Fundació ACE (ACE). The group of medical staff included doctors, nurses, psychologists, and therapists who were selected from the two centers based on qualification criteria, such as the experience with patients suffering from cognitive impairments. The potential user group included persons aged between 55 and 90 years with MCI or early stages of AD defined by MMSE score of 20-26 points. The third group in that selection was related to potential user groups, as these participants were responsible for taking care of the patients. In the case where many caregivers took care of a patient, the respondent group included the most involved and closest ones to the patients (e.g., family members).

Participants were randomly selected for the study basing only on the time of their arrival at the centers (LUM and ACE) during April-June 2015. In total, we received 264 completed questionnaires (100 from medical staff, 81 from caregivers, and 83 from potential users). One hundred fifty-four surveys ( 50 from medical staff, 51 from caregivers, and 53 from potential users) were gathered by ACE; the remaining 110 surveys were collected in Lublin (50 from medical staff, 30 from caregivers, and 30 from potential users). Table 1 presents the percentage values for gender distribution in the tested group. At least $2 / 3$ of respondents were women.

\begin{tabular}{|c|c|c|c|c|}
\hline & \multicolumn{4}{|c|}{ GENDER } \\
\hline & \multicolumn{2}{|c|}{ LUM } & \multicolumn{2}{|c|}{ ACE } \\
\hline & Male & Female & Male & Female \\
\hline Potential users & 10 (33.33\%) & 20 (66.67\%) & 21 (39.62\%) & 32 (60.38\%) \\
\hline Caregivers & $6(20 \%)$ & 24 (80\%) & 20 (40\%) & 30 (60\%) \\
\hline Medical staff & 12 (24\%) & 38 (76\%) & $8(16 \%)$ & 42 (84\%) \\
\hline
\end{tabular}


A brief characteristic of the group of cognitive impairment patients is shown in Table 2. Among potential users 16 (19.28\%) were $\mathrm{AD}$ patients and $67(80.72 \%)$ were $\mathrm{MCI}$ patients.

The issues included in the surveys, as well as their general layout, were similar for all stakeholders. The survey questionnaires included questions related to four sections: functional requirements; human-robot interaction, the design of the robotic assistant and social acceptance aspects.

The questionnaire layout was fully prepared by the authors of the survey and included close-ended and open-ended questions in each section. The data analysis shown in the tables are the results of the interpretation of the robot role from the stakeholders point of view. The data analysis shown in the Tables 1-6 was performed based on the answers given to closed-ended questions.

Based on the answers from close-ended questions, we specified prioritization of the stakeholders requirements and needs regarding robotic assistants. The requirement with high level priority means that a robotic assistant must have this function, medium level-should have, if possible, and low-not necessary for implementation.

- High priority-answers that were chosen by over $50 \%$ of the respondents and pointing to a more than average necessity of functional requirement.

- Medium priority answers marked by $25-50 \%$ of the respondents and pointing to average necessity of functional requirement.

- Low priority-answers chosen by less than $25 \%$ of the respondents and pointing to a less than average necessity of functional requirement.

The layout of the questionnaire has caused the necessity to use two methods of prioritization.

The first method of prioritization was performed using the Likert format of answers in surveys. It was done with data regarding the prioritization of different capabilities of robotic assistants with regard to human interaction, where stakeholders had a possibility to choose one out of five answers. It implies that the scores are valued as follows: 1 (very important, a patient has substantial difficulties with this and the proposed solution is desirable), up to 5 (very unimportant, a patient can do this on their own without any difficulty and the solution is

TABLE 2 | Brief characteristic of the group of cognitive impairment patients.

\begin{tabular}{lccccc}
\hline & \multicolumn{2}{c}{ Alzheimer disease } & & \multicolumn{2}{c}{ Mild cognitive impairment } \\
\cline { 2 - 3 } \cline { 5 - 6 } & LUM & ACE & & LUM & ACE \\
\hline Male & 3 & 7 & & 8 & 13 \\
Female & 4 & 12 & & 15 & 21 \\
Education level & Elementary (2) & Elementary (6) & Elementary (7) & Elementary (11) \\
& Secondary (4) & Secondary (10) & Secondary (12) & Secondary (17) \\
& Higher (1) & Higher (3) & Higher (4) & Higher (6) \\
Positive attitude & & & 81.01\% & \\
toward new & & & & & \\
technologies & & & & & \\
\end{tabular}

not desirable) (29). Next transformation based on the $\%$ from $1(80 \%+$ difficulty) to 5 ( $0 \%$ difficulty) was done After that the prioritization was conducted as follows: high priority mean less than 2, through medium priority between 3 and 2, up to low priority more/equal than 3 .

In the part devoted to identification of main situations when robotic assistants could be helpful, where stakeholders had opportunity to choose one of three answers, the answers were categorized into three groups: must, might, should not, and then prioritization was performed regarding to the frequency of answers according to general prioritization assumption described above.

Regarding prioritization of the way in which robotic assistants should be operated, simple analysis was performed based only on the frequency of answers according to general assumption.

In order to present accurately the differences in requirements for the robot between potential users, caregivers, and medical personnel, answers from both open-ended and close-ended questions were used (Table 7).

Visualization in diagrams was also used for the effective presentation of the outcomes.

\section{RESULTS}

\section{Focus Groups}

One of the main goals of the focus groups was to define a list of daily tasks for which a certain level of quality must be maintained or even improved by the robotic system and which are crucial for supporting life quality of potential users. Furthermore, general safety rules and social acceptance conditions of human-robotic assistant interaction were explored.

As the main problems and challenges in $\mathrm{MCI}$ and $\mathrm{AD}$ patient care the medical personnel highlighted problems with: regular medication intake, task accomplishment, and general forgetfulness.

In comparison to medical personnel, the caregivers focused on issues connected with daily care and hygienic procedures: dressing and changing clothes, proper nutrition, bathroom usage, and other daily activities like cooking, shopping, and laundry.

\section{Surveys}

\section{Social Acceptance Aspects}

Social acceptance of robotic assistants reached a high level among all groups of respondents. Most of them believe that a robot may be really helpful during daily life routines. Over $80 \%$ of the respondents think that it is good idea to replace a human caregiver with a robotic assistant. Only fewer than 20\% of respondents think that it is questionable, because robots never fully replace humans. More than $75 \%$ of caregivers agreed to leave the potential user alone with a robot. However, caregivers $(83,95 \%)$ similarly as potential users $(69,88 \%)$ need minimum 3-4 training sessions before they agree to cooperate with a robotic assistant.

Over $60 \%$ of potential users would like to treat robotic assistants as friends, which means that they would like to personalize it and show the robotic assistant to their family and friends. It is necessary for users to feel safe with a robotic assistant. That 
TABLE 3 | Prioritization of functional requirements.

\begin{tabular}{|c|c|c|c|c|c|c|c|c|}
\hline $\begin{array}{l}\text { The level of support offered by a robotic } \\
\text { assistant during activities of everyday life }\end{array}$ & $\begin{array}{l}\text { Frequency } \\
\text { of responds } \\
\text { (\%) }\end{array}$ & $\begin{array}{l}\text { Users } \\
\text { priority }\end{array}$ & $\begin{array}{l}\text { Frequency } \\
\text { of responds } \\
\text { (\%) }\end{array}$ & $\begin{array}{l}\text { Caregivers } \\
\text { priority }\end{array}$ & $\begin{array}{l}\text { Frequency } \\
\text { of responds } \\
(\%)\end{array}$ & $\begin{array}{l}\text { Medical } \\
\text { staff } \\
\text { priority }\end{array}$ & $\begin{array}{l}\text { Frequency } \\
\text { of responds } \\
\text { (\%) }\end{array}$ & $\begin{array}{l}\text { Overall } \\
\text { priority }\end{array}$ \\
\hline Calls for help, if something happens to the patient & 64.27 & $\mathrm{H}$ & 79.01 & $\mathrm{H}$ & 87.02 & $\mathrm{H}$ & 77.41 & $\mathrm{H}$ \\
\hline Detection of obstacles on the floor to prevent falls & 57.83 & $\mathrm{H}$ & 58.02 & $\mathrm{H}$ & 68.64 & $\mathrm{H}$ & 61.98 & $\mathrm{H}$ \\
\hline $\begin{array}{l}\text { Reminds the patient about boiling water, turning } \\
\text { off the gas and lights }\end{array}$ & 55.42 & $\mathrm{H}$ & 69.14 & $\mathrm{H}$ & 82.15 & $\mathrm{H}$ & 69.75 & $\mathrm{H}$ \\
\hline $\begin{array}{l}\text { Monitors correctness of the patient's medication } \\
\text { intake }\end{array}$ & 51.81 & $\mathrm{H}$ & 64.20 & $\mathrm{H}$ & 93.17 & $\mathrm{H}$ & 71.28 & $\mathrm{H}$ \\
\hline $\begin{array}{l}\text { Reminds the patient that it is time for him/her to } \\
\text { take his/her medication }\end{array}$ & 50.60 & $\mathrm{H}$ & 74.07 & $\mathrm{H}$ & 89.12 & $\mathrm{H}$ & 72.39 & $\mathrm{H}$ \\
\hline Provides cognitive exercise to the patient & 38.55 & M & 54.32 & $\mathrm{H}$ & 88.93 & $\mathrm{H}$ & 62.47 & $\mathrm{H}$ \\
\hline Finds things the patient is looking for & 51.81 & $\mathrm{H}$ & 43.21 & M & 45.16 & M & 46.65 & M \\
\hline $\begin{array}{l}\text { Is able to reach medication, which is difficult to } \\
\text { reach for the patient }\end{array}$ & 46.99 & M & 54.32 & $\mathrm{H}$ & 48.43 & M & 49.78 & M \\
\hline $\begin{array}{l}\text { Reminds the patient that it is time for his/her meal } \\
\text { or time to drink something }\end{array}$ & 24.50 & $\mathrm{~L}$ & 54.32 & $H$ & 67.15 & $\mathrm{H}$ & 49.80 & M \\
\hline $\begin{array}{l}\text { Stimulates the patient to keep in touch with family } \\
\text { and friends }\end{array}$ & 24.10 & $\mathrm{~L}$ & 53.09 & $\mathrm{H}$ & 62.19 & $\mathrm{H}$ & 47.42 & M \\
\hline $\begin{array}{l}\text { Reaches for fallen utensils and hands them over } \\
\text { to the patient, in order to prevent the patient from } \\
\text { bending over. Grasps things from the floor/shelves }\end{array}$ & 45.78 & M & 38.27 & M & 44.13 & M & 42.85 & M \\
\hline Provides physical exercises to the patient & 39.76 & M & 43.21 & M & 63.22 & $\mathrm{H}$ & 49.70 & M \\
\hline $\begin{array}{l}\text { Reminds about important dates such a birthdays } \\
\text { and medical appointments }\end{array}$ & 42.17 & M & 41.98 & M & 61.29 & $\mathrm{H}$ & 49.35 & M \\
\hline $\begin{array}{l}\text { Robotic assistant recognizes strangers and informs } \\
\text { family members about such visits }\end{array}$ & 40.02 & M & 61.14 & $H$ & 81.88 & $\mathrm{H}$ & 62.36 & $H$ \\
\hline $\begin{array}{l}\text { Recognizes when it can or cannot open the house } \\
\text { door }\end{array}$ & 39.76 & M & 60.49 & $\mathrm{H}$ & 80.01 & $\mathrm{H}$ & 61.37 & $\mathrm{H}$ \\
\hline Helps the patient prepare food & 24.30 & $\mathrm{~L}$ & 23.46 & $\mathrm{~L}$ & 65.17 & $\mathrm{H}$ & 39.52 & M \\
\hline Helps the patient put on clothes & 24.91 & $L$ & 32.10 & M & 43.26 & M & 34.07 & M \\
\hline Helps the patient take on/off her/his shoes & 22.89 & $L$ & 29.63 & M & 51.36 & $\mathrm{H}$ & 35.74 & M \\
\hline Helps the patient with a shopping list & 21.69 & $L$ & 24.57 & $\mathrm{~L}$ & 17.52 & $\mathrm{~L}$ & 20.99 & $L$ \\
\hline Reminds the patient about TV programs & 16.87 & $\mathrm{~L}$ & 24.69 & $\mathrm{~L}$ & 22.13 & $\mathrm{~L}$ & 21.26 & $\mathrm{~L}$ \\
\hline Helps the patient to clean the house & 25.37 & M & 24.63 & $\mathrm{~L}$ & 24.79 & $\mathrm{~L}$ & 24.92 & $\mathrm{~L}$ \\
\hline Helps the patient to put her/his feet on the footrest & 12.05 & $\mathrm{~L}$ & 30.86 & M & 11.87 & $\mathrm{~L}$ & 17.75 & $\mathrm{~L}$ \\
\hline
\end{tabular}

TABLE 4 | Prioritization of the way in which robotic assistants should be operated.

\begin{tabular}{|c|c|c|c|c|c|c|c|}
\hline $\begin{array}{l}\text { The way robotic assistants } \\
\text { should be operated }\end{array}$ & $\begin{array}{c}\text { Percentage of positive } \\
\text { responses (\%) }\end{array}$ & $\begin{array}{l}\text { Users } \\
\text { priority }\end{array}$ & $\begin{array}{l}\text { Percentage of positive } \\
\text { responses }(\%)\end{array}$ & $\begin{array}{l}\text { Caregivers } \\
\text { priority }\end{array}$ & $\begin{array}{c}\text { Percentage of positive } \\
\text { responses (\%) }\end{array}$ & $\begin{array}{l}\text { Medical staff } \\
\text { priority }\end{array}$ & $\begin{array}{l}\text { Overall } \\
\text { priority }\end{array}$ \\
\hline $\begin{array}{l}\text { By simple voice commands } \\
\text { (voice operated system) }\end{array}$ & 61.45 & $\mathrm{H}$ & 85.19 & $\mathrm{H}$ & 89.57 & $\mathrm{H}$ & $\mathrm{H}$ \\
\hline By touch screen & 30.12 & M & 32.10 & M & 47.14 & M & M \\
\hline By simple gestures & 27.71 & M & 28.40 & $M$ & 64.31 & $\mathrm{H}$ & M \\
\hline By a remote control & 25.30 & $M$ & 27.16 & M & 31.16 & $\mathrm{M}$ & M \\
\hline By keyboard/buttons & 3.61 & $\mathrm{~L}$ & 7.41 & $L$ & 9.19 & $\mathrm{~L}$ & $\mathrm{~L}$ \\
\hline $\mathrm{N} / \mathrm{A}$ & 12.05 & & 3.70 & & 5.17 & & $\mathrm{~L}$ \\
\hline
\end{tabular}

is why $73.5 \%$ of respondents wish the robotic assistant was able to call for help if something bad happens to them and even $53 \%$ of them would be ready to move some furniture to let a robotic assistant move everywhere. It is also important for them whether the robotic assistant has a stand-by function even during the night. 


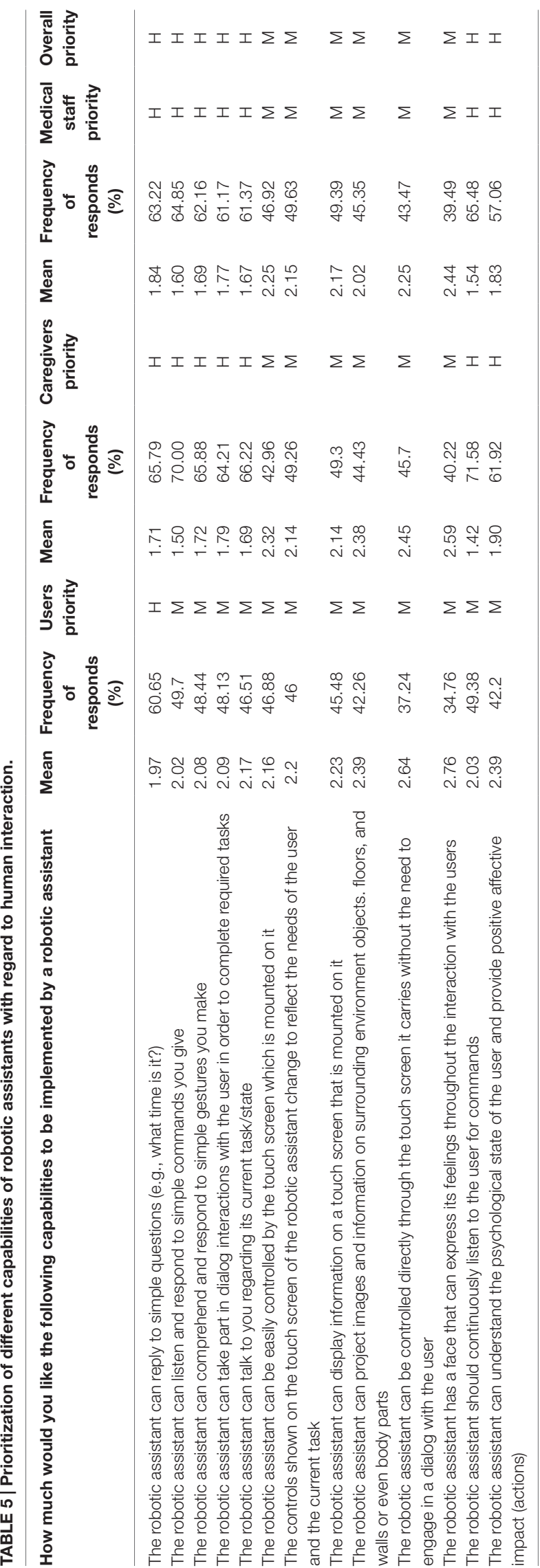

TABLE 6 | Percentage distribution of respondents' preferences regarding to height of the robot.

\begin{tabular}{llccc}
\hline $\begin{array}{l}\text { Height of robotic } \\
\text { assistant }\end{array}$ & & $\begin{array}{c}\text { Potential } \\
\text { users (\%) }\end{array}$ & $\begin{array}{c}\text { Caregivers } \\
\text { (\%) }\end{array}$ & $\begin{array}{c}\text { Both } \\
\text { groups (\%) }\end{array}$ \\
\hline Shorter than me: & Knee-high & 3.61 & 3.70 & 3.66 \\
$59.15 \%$ & Waist-high & 19.28 & 20.99 & 20.12 \\
& Chest-high & 34.94 & 35.80 & 35.37 \\
Taller than me: & Up to $20 \mathrm{~cm}$ & 6.02 & 4.94 & 5.49 \\
$5.49 \%$ & $\begin{array}{l}\text { More than } 20 \mathrm{~cm} \\
\text { The same height }\end{array}$ & - & - & - \\
& as me & & 27.16 & 23.17 \\
& \multicolumn{1}{c}{$\mathrm{N} / \mathrm{A}$} & 16.87 & 3.70 & 12.19 \\
\hline
\end{tabular}

\section{Functional Requirements}

As shown in Table 3, in order to define the overall priority level of each requirement, the priority levels that were attributed to them by each group involved in the surveys were taken into account. Functional requirements are categorized as high, medium, and low priority. The first seven functionalities listed in the table have high priority for the respondents. These functionalities are especially associated with the potential user safety, like calling for help if something happens to the patient or with general memory support by reminding the users about medication intake or about boiling water, turning off the gas and light.

Functions with medium priority regarding basic daily activities include preparing food and dressing. Also, robotic assistants might be helpful in reaching, grasping, finding things, and reminding of task ordering, which helps cognitive impairment patients compensate for their shortcomings.

Low priority functionalities regarding entertainment, relaxation and shopping.

\section{Proactively (Autonomously) vs. on Demand}

Memory alteration significantly decreases the quality of life in many aspects of daily living. In order to reduce the disease progress of $\mathrm{MCI}$ and $\mathrm{AD}$ patients, medical personnel emphasized that most of everyday life activities should be done independently. That is why robotic assistants should motivate and encourage the primary user to stay active. However, we cannot forget about the safety of the users, which is why in specific potentially hazardous situations robotic assistant must be able to take action autonomously, e.g., prevent falls (detect obstacles) or in the case of operating electrical and gas devices without the user's attention. General findings show that the functions implemented into the robotic assistant and the ones that are correlated with the patient's safety must be performed autonomously. At times, a potential user might not be able to do something alone (loss of consciousness) or may not realize or just forget that something important (e.g., medications intake) must be done.

Both groups (medical staff and caregivers) agreed that in unexpected and serious events such as falls, loss of consciousness, a robotic assistant should autonomously alarm relevant services and relatives.

Activities that should be done exclusively on demand are: carrying heavy things, preparing food, reminding about important 
TABLE 7 | The differences in approach to robot functionalities in which robot must be equipped between caregivers, potential users, and medical staff.

\begin{tabular}{|c|c|c|}
\hline Medical staff & Caregivers & Users \\
\hline $\begin{array}{l}\text { - Informs about the danger arising from improper object location, } \\
\text { - Reaches for fallen utensils and hands them over to the patient, } \\
\text { in order to prevent the patient from bending over, } \\
\text { - Register of falls, } \\
\text { - Reminds about returning to household tasks after being interrupted, } \\
\text { - Suggests which tasks are prioritized, } \\
\text { - Fetches things patient asks for, } \\
\text { - Stimulates the patient to keep in touch with family and friends, } \\
\text { - Reminds about the time for preparing meals, } \\
\text { - Suggests appropriate diet, } \\
\text { - Helps the patient prepare food, } \\
\text { - Informs family members about visits, } \\
\text { - Explains how to perform cognitive and physical exercises, } \\
\text { - Increase/decrease the difficulty of cognitive exercises. } \\
\text { - Reminds about technical aids that improve user mobility } \\
\text { - Provides instructions on how to use medical equipment } \\
\text { - Monitors proper selection of clothes, } \\
\text { - Points out improper selection of clothes, } \\
\text { - Helps in dressing up, } \\
\text { - Remembers where important items were placed, } \\
\text { - Reminds about events/deadlines. }\end{array}$ & $\begin{array}{l}\text { - } \text { Calls for help, if something happens to the patient, } \\
\text { - } \text { his/her medication, } \\
\text { - Monitors the correctness of the patient's medication } \\
\text { intake, } \\
\text { - Detects obstacles on the floor to prevent falls, } \\
\text { - Reminds the patient about boiling water, turning off the } \\
\text { gas and lights, } \\
\text { - Is able to reach medication, which is difficult to reach } \\
\text { for the patient, } \\
\text { - Stimulates the patient to keep in touch with family and } \\
\text { friends, } \\
\text { - Reminds the patient that it is time for his/her meal or } \\
\text { time to drink something, } \\
\text { - Recognizes when it can or cannot open the house } \\
\text { door, }\end{array}$ & $\begin{array}{l}\text { - Calls for help, if something } \\
\text { happens to the patient, } \\
\text { - Reminds the patient that it is } \\
\text { time for him/her to take his/her } \\
\text { medication, } \\
\text { - Monitors correctness of the } \\
\text { patient's medication intake, } \\
\text { - Detects obstacles on the floor to } \\
\text { prevent falls } \\
\text { - Reminds the patient about boiling } \\
\text { water turning off the gas and } \\
\text { lights, } \\
\text { - Finds things the patient is looking } \\
\text { for. }\end{array}$ \\
\hline
\end{tabular}

dates and medical appointments, dressing, turning off a TV/ radio, and reading a book. These functionalities or activities do not have direct influence on the potential user's health and safety.

\section{Human-Robot Interaction}

Table 4 shows prioritization of the types of human-robot interaction. As a result of the analysis regarding the mode of human-robot assistant interaction, a "high priority" level was assigned to voice-operated system. It seems reasonable to expect it because it is a human natural and simplest way of communication. Medium priority was assigned to simple gestures, remote control, and touch screen. These ways of communication are widespread in new technology of electronic devices like TV sets, smartphones, tablets, etc.

The way of interaction is one of the most important factors influencing the degree of robot acceptance. Table 5 presents prioritization of different capabilities of a robotic assistant in relation to human interaction. It is important to all respondents that potential user may interact with robotic assistant, it means that:

- the robotic assistant can reply to simple questions,

- the robotic assistant can listen and respond to simple commands given by the user,

- the robotic assistant can comprehend and respond to simple gestures made by the user,

- the robotic assistant can take part in dialog interactions with the user in order to complete required tasks,

- the robotic assistant should continuously listen to the user for commands,

- the robotic assistant can talk back to the user regarding its current task/state,

- the robotic assistant can understand the psychological state of the user and provide positive affective impact (actions).

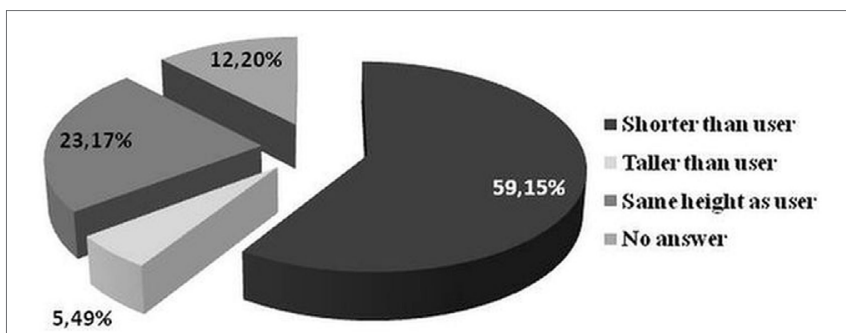

FIGURE 1 | Percentage distribution of respondents' preferences regarding the height of the robot.

\section{Design of the Robotic Assistant}

The majority of the respondents would like a robotic assistant to have an anthropomorphic appearance (woman or neutral) and a face with positive emotional expressions. The material from which the robotic assistant will be made does not matter for the respondents.

Most of the respondents think that the robotic assistant should be shorter than the user and the best height is approximately chest height. Table $\mathbf{6}$ and Figures $\mathbf{1}$ and $\mathbf{2}$ show the percentage distribution of respondents' preferences regarding to height of the robot.

\section{Differences in Prioritization among All Groups of Respondents}

As it is shown in Table 7, there are some requirements important for all groups of respondents. They are associated with the safety of users during daily routine activities. Potential users belong to the group with the fewest number of requirements, which must be implemented into a robot (high priority functionalities). They indicated only six high priority functionalities opposite to 10 pointed by caregivers and 20 important functionalities listed 


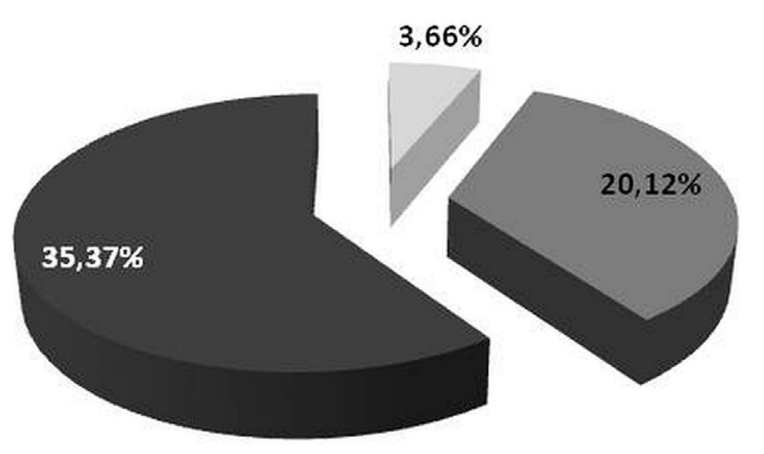

Knee-high $\square$ Waist-high $\square$ Chest-high

FIGURE 2 | Percentage distribution of prevailing answers from Figure 1.

TABLE 8 | The differences in prioritization among Polish and Spanish respondents regarding functionalities of robotic assistant.

\begin{tabular}{|c|c|c|c|c|}
\hline \multirow[t]{2}{*}{ Functionalities } & \multicolumn{4}{|c|}{ Prioritization of functionalities } \\
\hline & $\%$ & Poland & $\%$ & Spain \\
\hline $\begin{array}{l}\text { Stimulating the patient to keep in touch with } \\
\text { family and friends }\end{array}$ & 20.64 & Low & 38.10 & Medium \\
\hline Finding things the patient is looking for & 24.71 & Low & 53.95 & High \\
\hline $\begin{array}{l}\text { Reminding about important dates such a } \\
\text { birthday and medical appointments }\end{array}$ & 19.68 & Low & 51.69 & High \\
\hline $\begin{array}{l}\text { Reaching for fallen utensils and handing } \\
\text { them over to the patient in order to prevent } \\
\text { the patient from bending over. Grasping } \\
\text { things from the floor/shelves }\end{array}$ & 23.25 & Low & 50.92 & High \\
\hline Helping the patient clean the house & 17.00 & Low & 38.18 & Medium \\
\hline $\begin{array}{l}\text { Helping the patient properly button her/his } \\
\text { clothes }\end{array}$ & 17.89 & Low & 31.86 & Medium \\
\hline Helping the patient take off her/his shoes & 23.01 & Low & 32.72 & Medium \\
\hline $\begin{array}{l}\text { Helping the patient put her/his feet on a } \\
\text { footrest }\end{array}$ & 16.85 & Low & 26.54 & Medium \\
\hline Fetching things, the patient asks for & 19.68 & Low & 39.69 & Medium \\
\hline Helping the patient draw up a shopping list & 12.95 & Low & 33.47 & Medium \\
\hline Providing physical exercises for the patient & 32.18 & Medium & 50.40 & High \\
\hline
\end{tabular}

by medical staff. The paternalistic approach of caregivers and medical personnel regarding the functionalities that must be implemented in the robotic assistant is the result of fears raised in open-ended questions as to whether the robot will be able to fully support human caregiver. They need to be sure that there are no doubts about leaving the patient alone with the robot. What is more, the medical staff would like the robot to possess functionalities, which might slow down or even induce a decrease in cognitive impairment of potential users.

\section{Differences between Polish and Spanish Respondents}

There are some differences in prioritization among Polish and Spanish respondents regarding functionalities. The most noticeable differences are shown in Table 8.
All the functionalities listed in the table above and implemented into the robot have higher priority for the Spanish respondents than the Polish ones. Survey analyses show that the Polish respondents have lower expectations regarding the robotic assistant than their counterparts from Spain.

\section{DISCUSSION}

This study, using a multimodal approach (focus groups, survey questionnaire) among three groups: older people with $\mathrm{AD}$ and early dementia stages, caregivers and medical staff, determined the desired requirements of the cognitively impaired patient, which may be met by a robotic assistant and would be enough to introduce robots as home care assistants. In this sections, the findings are discussed with respect to their levels of priority and with reference to the literature.

The most important capability of assistive technology is to handle emergencies in a private home. Robotic assistants have to recognize life-threatening situations by ongoing monitoring of health parameters, detecting falls and detecting dangerous situations in the household environment (e.g., working electrical or gas appliances). It is also important that robotic assistant must be able to inform appropriate support units and relatives in the case of emergency situation. Emergency alarms are a key point of robot safety, especially for social robots, since these robots aim to prevent any critical event for older people $(8,14,30)$.

In this respect, the management of critical situations is one of the most preferred functionalities. The most common sources of home accidents leading to morbidity and mortality are falls and cardiovascular diseases (31-33). Along the same line of reasoning, our participants identified that a high level of priority, as a safety consideration, should be assigned to the removal of clutter and obstacles that may pose trip hazard.

Moreover, it is very important that robots are able to detect dangerous home environment situations regarding working electrical or gas appliances left uncontrolled. People with dementia who are living at home demonstrated that those living alone were perceived to be more at risk than those living with someone, and the most commonly reported risk is the one of fire (34).

People with dementia are at risk from self-neglect, which means that vulnerable adult is unable to exercise basic self-care (25). Signs of self-neglect include dehydration, malnutrition, untreated medical conditions, poor personal hygiene, unsafe or unsanitary living conditions, inappropriate or inadequate clothing, and inadequate housing or homelessness (35). ${ }^{2}$ In this context, the essential strategy that is assigned to the robot is to prevent self-neglect on the part of people suffering from dementia.

The tasks of reminding about and monitoring medication intake were mentioned as high level priority functionalities, which must be implemented into the robotic assistant. Douglas et al. emphasized that accidental injuries from errors in drug self-administration are more likely to occur for people with dementia than injury from fires/burns and wandering. These

${ }^{2}$ https://ncea.acl.gov/faq/. 
errors were attributed to cognitive deficits, sensory or physical problems with dispensers, or the complexity of the treatment regimen (31).

Interesting findings were recorded with regard to questions about reminding of meal or drink times and keeping in touch with family and friends. Elderly people with cognitive impairment reported that these functionalities are not necessary to be implemented. On the contrary, caregivers and medical staff argued that the robots must have the ability to perform these tasks. The differences may be a result of varied perceptions of disability. It is hard for early dementia patients to agree that they must be reminded about the simplest and basic human needs, which for older adults can be found as associated with the idea of "giving up" or of admitting defeat (36).

An important aspect of ensuring home security for persons with cognitive impairment is to prevent visits of unknown persons. It is especially important to avoid intrusions into the household. Older people, especially those with cognitive impairment, are an easy target for thieves. Hence, recognizing strangers is such an important functionality for caregivers and medical staff with regard to ensuring user safety.

Regarding the functionalities such as finding and reaching for things to bring them to patients, they are highly important for the primary users and of medium importance for the caregivers and medical staff. It is a very convenient feature, which makes life easier but otherwise may lead to the reduction of stimulating patients to remember. The same finding was observed in other studies where cognitively impaired patients were also interested in the functionalities offered by an assistive robot, like the object finding system (12).

These functionalities for monitoring and supervising the health and safety of the user are at a high level of priority and should be provided autonomously.

The other important ability of the robot is to provide cognitive stimulation to the potential user in two ways.

First, by engaging in physical and psychological activity and second, by providing cognitive entertainments. According to the medical personnel and caregivers' opinions, this ability should be provided proactively but the potential users' opinions were divided with a proportion 60/40 in the direction of ondemand. Recent research has been increasingly focused on the cognitive system, which is necessary to boost human cognitive capabilities or at least to inhibit the progress of cognitive decline (37-39).

Furthermore, traditional cognitive training with paper and pencil usually requires experienced instructors (40), but those qualified instructors may be unavailable for all in need in the coming years. For these reasons, opportunities for communication with others often decrease with advanced age and poor communication environment is linked to impaired cognitive function in the elderly (41). Thus, robotic assistance is a novel strategy to improve cognitive functions and prevent cognitive decline.

Communication is essential to maintain motivation (42).

Living with a human-type communication robot may not only improve cognitive functions but also provide beneficial outcomes for daily activities, morbidity, and mortality in the elderly (38).
Communication based on voice commands was chosen as a high priority and the simplest way of human-robot interaction. This type of communication is natural for man and it requires no additional effort in this field. Respondents indicate that robotic assistants must be able to talk in response to the user regarding its current task/state and should also continuously listen to the user for commands. Reciprocity is an important factor in human-human interaction, so it can be expected that it should also play a major role in human-robot interaction (43). It is very important that potential user and robot engage not only in natural interaction but also in alternative means of non-verbal communication such as simple gestures, remote control, and touch screen, which were chosen. Our findings are in line with previous studies (44). Additionally, Frennert et al. identified that for some users subtitles should be displayed on the screen while the robot is speaking. This solution would help to avoid misunderstandings when the robot is speaking and the robot's voice is hardly audible.

We also found positive attitude toward socio-emotional interaction between the elderly and robots, which was expressed in a request that the robotic assistant should be able to understand the psychological state of the user and then provide positive affective impact. Socio-emotional interaction poses key requirements with which to create sustainable relationships between the elderly and robots, and this type of interaction will enhance the users' acceptance and encourage the adoption of the assistive robotic system (45). The feeling of loneliness may negatively impact a person's ability to act and engage in everyday activities. Robotic assistants could be a substitute for reducing loneliness of elderly people in two ways with direct and indirect impact. The direct influence means positive emotional human-robot interaction, or the robot as a friend. The indirect effect can be provided by stimulating the user to keep in touch with family and friends. Svanstrom et al. found that without the presence of others, a person with dementia seemed to lack initiative and experienced difficulties in managing everyday life. The authors highlight the importance of the presence of caregivers for those who live alone with dementia, who address the needs for socialization and do not solely focus on specific tasks or physical needs (46). In our study part of caregivers confessed that the burden of caring for their relatives and unmet needs themselves provoke frustration and unfriendly attitude toward their relative. They considered a robotic assistant to be a solution to improve relationships with their relative. This is a very important aspect, which must be considered because maintaining toxic relationship when the supporting person becomes frustrated may terminate a caregiving relationship (25).

Considered less important, the functionalities, which were assigned medium level priority were connected with performing basic daily activities, such as preparing food, dressing. They considered that robots might be helpful by reaching, grasping, finding things, and maintaining the sequence of tasks, which helps them compensate for their impairment. This result is not surprising, as demonstrated in many previous studies $(44,47,48)$.

Surveys also identified robot capabilities with low importance for the Polish respondents. They are related to daily relaxation activities, like reminding about TV programs or 
these relating to assistance in shopping and cleaning the house. However, interesting findings were observed when comparing Polish and Spanish respondents. The functionality of cleaning the house was identified as high-value for Spanish respondents contrary to the Polish ones. Also, other functionalities listed above, which are not important for Polish users, are more important for Spanish ones. Here, the national and cultural differences are the most visible, which may stem from different lifestyles. Research performed on populations from well developed countries indicated that the perception of the robot as enjoyment or entertainment increases the inclination to use it $(49,50)$.

Another aspect of the requirements for the robot is its appearance. According to the literature, the robot's appearance must be appropriately correlated with its functionality. It influences how people appraise the capabilities of the robot. It means that if a robot looks like a toy or pet, it will be treated as a source of entertainment and nobody will expect that such a robot is responsible for monitoring human health (51). On the other hand, humanoid robots are also undesirable $(11,52,53)$. Summarizing, it is unacceptable and may finally lead to a market failure of a robot when its appearance is not consistent with its functionality. That is why it was important for us to find our target group expectations about robot capabilities or functionalities with regard to its appearance. The results of our surveys show that the majority of the respondents would like a robotic assistant to have an anthropomorphic appearance (woman or neutral) with positive emotionally expressive face. Most of the respondents think that the robotic assistant should be shorter than the user and the best height is approximately chest height. It confirms findings from other studies where most of the respondents would like the robot to have neutral/genderless (44) or more realistic sophisticated feminine appearance, which is more appropriate in expressing basic emotions (54).

The main issue in developing new robot is its acceptance. If cooperation of people with robots is to be successful, they need to be accepted by the target group. Even the best robot with many functionalities but without social acceptance is worthless. According to one of the definitions (55), acceptance may be described as the willingness to incorporate the robot into personal life. So, it is subjective users' perceptions of what robots are, how they work, and what they can or cannot do that will determine how they are perceived and finally accepted (56). Many of individual factors such as age, gender, education, cognitive ability, culture, needs, experience with technology, and the level of anxiety affect the acceptability of robots (57).

In the general context of the acceptance of robots and of the prioritization of the user requirements in relation to robotic assistants, we did not find differences in terms of age and gender. In the literature, we can find that the willingness to use robots by elderly people depends on the context. When people were asked whether they could imagine living on a daily basis with robots, the most positive responses were from young adults and the least positive from adults over 65 years. On the other hand, when asked the question in a different way whether they would accept a robot to help them gain independence when they could no longer handle everyday tasks, acceptance increased particularly in the oldest age range $(52,57)$. In the same line of reasoning, we noted that over $80 \%$ of the respondents accept robotic assistants as a way to remain healthy and safe in their own homes. The high level of acceptance in our study also confirmed the findings that the acceptability of this specific solution is probably influenced by the coping strategies $(58,59)$, which is a matter of perceived self-efficacy. Pino et al. (49) and Bandura (60) demonstrated that participants with MCI and caregivers had more positive perception of the usefulness of robots than healthy older adults. Cesta et al. emphasized that it is important to address the issue of how frailty is perceived, with reference to both health in general and fear of cognitive weakening. More specifically, it can influence the evaluation of potential aid in everyday life, namely the robotic assistant. In the literature, it can be found that MCI persons did not consider themselves as potential users. They stated that a robot could be potentially useful either for themselves in the future or for other older adults suffering from frailty, loneliness, and disability $(12,36,61)$.

The differences in the readiness to use a robotic assistant between elderly people are noticeable. Some people want to be independent and not be a burden to other people, and for them, a robot would be an ideal solution. This group of elderly people expresses readiness to accept a robotic assistant to help them (62). Others are now convinced that they do not need or want a robot. They considered that it is the duty of the family, the state to provide them with constant care, and not leaving them in the care of a robot. This attitude could be considered as passive resistance $(12,63)$. Many studies indicated possible factors underlying this attitude (12, 63-66).

On the whole, this attitude is associated with the type of personality and sometimes with a strong sense of fear of something new. In our study, above $80 \%$ of respondents accept a robot as a caregiver but being presently ready to remain in the care of a robot is expressed by only half of them. Among our respondents, it can be observed that postponed readiness probably stems from the lack of familiarity with technology, which can be explained by the fact that almost $70 \%$ cognitively impaired respondents and almost $84 \%$ of caregivers expressed the need to attend training sessions (minimum 3-4 training sessions) before they agree to leave the users alone with a robotic assistant. Lack of familiarity with technology can be a reason for people to feel uncertain about robots $(67)$.

Direct experience of the usefulness of assistive devices can change older people's attitudes from considering assistive devices unnecessary to considering them very useful (68). Promotional strategies that present a product as comfortable and facilitating the life of the consumer will cause people to want it and consequently feel that these are needed. A good example of such a strategy is presented by companies introducing new innovative products to the market. Referring to the robot, considering that people tend to have limited knowledge, it is good to show them the robot's functionalities and how it can help them in daily activities, as it has been demonstrated by Broadbent et al. (57), who showed that, in individuals, unfamiliar with robotic healthcare, promoting appropriate expectations may be both feasible and effective. 
Acceptability has multidimensional aspects and requires a broad approach but may be modified. An interesting finding was observed during one study where, at first, most of the participants in the focus groups were negatively disposed toward the idea of robots but after $1 \mathrm{~h}$ or so, after finding out more about the robots, some of them changed their mind and thought robots might be "good for others but not themselves" (44). Koay et al. found peoples' preferences regarding robots changed over a period of time during a 5-week trial as participants grew familiar with robots (69).

The strength of our study is that the results were obtained from two autonomic centers and with the use of mixed methods with a wide range of stakeholders. Broadbent et al. emphasized that, to adjust the design of assistive robots, one has to assess the expectations and needs of a wide-range stakeholders. Most studies conducted in the dementia care context have only focused on one stakeholder group (21). Other included more group perspectives but involved a small sample size (49). A small sample size reduces both the chance of detecting true effects and the likelihood that a statistically significant result reflects a true effect (70). The minimum sample size for using maximum likelihood estimation is 100 (71) and this requirement was fulfilled in. our study (264 of the respondents). Additionally, the collected data came from different countries and provided an opportunity to identify different social cultural aspects in this field of study. This assisted researchers in identifying insights from different socio-cultural points of view (72).

It is worth mentioning that for the purpose of data, gathering focus groups were conducted. This type of methodology is considered an appropriate technique for preliminary data gathering; it makes it possible to obtain a wider range of experiences and ideas $(73,74)$. The selected groups held discussions based on their knowledge where appropriate. In the general context of dementia, caregivers and medical staff have the greatest knowledge to establish needs of individuals with cognitive impairment in a multidimensional aspect.

Two types of questions in surveys were used like closed-ended and open-ended. This methodology avoids bias because using only closed-ended question might force one to choose some response items even if respondents do not support exactly what the author thinks (12).

The main limitation of this study lies in the conceptual perception of an assistive robot rather than actual use in a sample of elderly people with cognitive impairment.

\section{CONCLUSION}

It will be a long time before a robot can be capable of supporting multiple activities in a physical manner in the home of an elderly person in order to enhance their independent living capacity. The results from our study might contribute to a better understanding of the users' needs and system requirements for the development of a robot intended to support older adults with cognitive impairment at home and their informal caregivers.
The development of robotic assistants in the general context of dementia requires both the understanding of the needs of the persons with cognitive impairment and the intensification of dissemination activities to shape a positive image of assistive robots.

In the literature, we can observe how the attitude toward technology has changed in the direction of adopting new solutions and perceiving them as something useful, pleasant. So, this phenomenon of positive change should gain pace in the direction of robotics seen as something natural and not stigmatizing people with infirmities.

Our results hold a promise that people with cognitive impairment are increasingly willing to take the robot to their home. Thus, assistive robots must meet the challenges as a caregiver of proven reliability, usability, and efficiency.

In view of the observations presented above, this study allows us to conclude that early dementia persons are increasingly willing to entrust themselves to the care of a robot that will meet the needs they have specified.

Nevertheless, longitudinal studies in the application of assistive robots are required.

\section{ETHICS STATEMENT}

Local ethics committee of Medical University of Lublin. Local ethics committee of Fundacio ACE. Project ethics advisory board.

\section{AUTHOR CONTRIBUTIONS}

AK-substantial contributions to the conception or design of the work, analysis, or interpretation of data for the work, drafting the work, and final approval of the version to be published. SS-interpretation of data for the work, revising it critically for important intellectual content, and final approval of the version to be published. CR, NT, JH-F, JR, US, KG-A, DS-S-design of the work, revising it critically for important intellectual content, and final approval of the version to be published. KR - substantial contributions to the conception revising it critically for important intellectual content and final approval of the version to be published. All authors agreed to be accountable for all aspects of the work in ensuring that questions related to the accuracy or integrity of any part of the work are appropriately investigated and resolved.

\section{ACKNOWLEDGMENTS}

We would like to thank Dr. Justyna Gerłowska for her contribution to our work. We would also like to thank all the RAMCIP partners.

\section{FUNDING}

This study was supported by European Commission under Horizon 2020 (European Union Research and Innovation programme. Grant number: 643433). 


\section{REFERENCES}

1. WHO. Word Health Organization. (2007). Available from: http://www.who. int/mediacentre/factsheets/fs362/en/

2. Yeh YC, Lin KN, Chen WT, Lin CY, Chen TB, Wang PN. Functional disability profiles in amnestic mild cognitive impairment. Dement Geriatr Cogn Disord (2011) 31:225-32. doi:10.1159/000326910

3. de Rotrou J, Wu YH, Hugonot-Diener L, Thomas-Antérion C, Vidal JS, Plichart M, et al. DAD-6: a 6-ltem version of the disability assessment for dementia scale which may differentiate Alzheimer's disease and mild cognitive impairment from controls. Dement Geriatr Cogn Disord (2012) 33:210-8. doi:10.1159/000338232

4. Alzheimer's Association. Alzheimer's disease facts and figures. Alzheimers Dement (2012) 8:131-68. doi:10.1016/j.jalz.2012.02.001

5. Bleiler L, Thies W. Alzheimer's disease facts and figures. Alzheimers Dement (2012) 8:131-68. doi:10.1016/j.jalz.2012.02.001

6. United Nations, Department of Economic and Social Affairs, Population Division. World Population Prospects: The 2006 Revision, Highlights, Working Paper No. ESA/P/WP.202. New York: United Nations (2007). Available from: http://www.un.org/esa/population/publications/wpp2006/WPP2006_ Highlights_rev.pdf

7. Kramer SC, Friedmann E, Bernstein PL. Comparison of the effect of human interaction, animal-assisted therapy, and AIBO-assisted therapy on longterm care residents with dementia. Anthrozoos (2009) 22(1):43-57. doi:10. 2752/175303708X390464

8. Moyle W, Jones C, Cooke M, Siobhan O, Sung B, Drummond S. Connecting the person with dementia and family: a feasibility study of a telepresence robot. BMC Geriatr (2014) 14:7. doi:10.1186/1471-2318-14-7

9. Peca A, Simut R, Pintea S, Costescu C, Vanderborght B. How do typically developing children and children with autism perceive different social robots? Comput Hum Behav (2014) 41:268-77. doi:10.1016/j.chb.2014.09.035

10. Šabanović S, Chang WL, Bennett CC, Piatt JA, Hakken D. A robot of my own: participatory design of socially assistive robots for independently living older adults diagnosed with depression. In: Salvendy JZG, editor. Human Aspects of IT for the Aged Population Design for Aging. Berlin, Germany: Springer (2015). p. 104-14.

11. Wu YH, Fassert C, Rigaud AS. Designing robots for the elderly: appearance issue and beyond. Arch Gerontol Geriatr (2012) 54(1):121-6. doi:10.1016/j. archger.2011.02.003

12. Wu YH, Cristancho-Lacroix V, Fassert C, Faucounau V, de Rotrou J, Rigaud AS. The attitudes and perceptions of older adults with mild cognitive impairment toward an assistive robot. J Appl Gerontol (2016) 35(1):3-17. doi:10.1177/ 0733464813515092

13. Bemelmans R, Gelderblom GJ, Jonker P, De Witte L. Socially assistive robots in elderly care: a systematic review into effects and effectiveness. J Am Med Dir Assoc (2012) 13(2):114-20.e1. doi:10.1016/j.jamda.2010.10.002

14. Fischinger D, Einramhof P, Papoutsakis K, Wohlkinger W, Mayer P, Panek P, et al. Hobbit, a care robot supporting independent living at home: firstprototype and lessons learned. Robot Auton Syst (2014) 75(Pt A):60-78. doi:10.1016/j. robot.2014.09.029

15. Libin A, Libin E. Robots who care: robotic psychology and robotherapy approach. Paper Presented at the Caring Machines: AI in Eldercare: Papers from the AAAI Fall Symposium. Technical Report: FS-05-02. Pittsburgh (2005).

16. Chu MT, Khosla R, Khaksar SM, Nguyen K. Service innovation through social robot engagement to improve dementia care quality. Assist Technol (2016) 11:1-11. doi:10.1080/10400435.2016.1171807

17. Arkin RC, Scheutz M, Tickle-Degnen L. Preserving dignity in patient caregiver relationships using moral emotions and robots. Paper Presented at the 2014 IEEE International Symposium on Ethics in Science. Chicago, IL: Technology and Engineering (2014). p. 1-5.

18. Pfadenhauer M, Dukat C. Robot caregiver or robot-supported caregiving? Int J Soc Robot (2015) 7:393-406. doi:10.1007/s12369-015-0284-0

19. Salichs MA, Encinar IP, Salichs E, Castro-González Á, Malfaz M. Study of scenarios and technical requirements of a social assistive robot for Alzheimer's disease patients and their caregivers. Int J Soc Robot (2015) 8(1):85-102. doi:10.1007/s12369-015-0319-6

20. Huschilt J, Clune L. The use of socially assistive robots for dementia care. J Gerontol Nurs (2012) 38:15-9. doi:10.3928/00989134-20120911-02
21. Mordoch E, Osterreicher A, Guse L, Roger K, Thompson G. Use of social commitment robots in the care of elderly people with dementia: a literature review. Maturitas (2013) 74:14-20. doi:10.1016/j.maturitas.2012.10.015

22. Scassellati B. How social robots will help us to diagnose, treat, and understand autism. In: Thrun S, Brooks R, Durrant-Whyte H, editors. Robotics Research. (Vol. 8), Berlin, Germany: Springer (2007). p. 552-63.

23. Vallor S. Carebots and caregivers: sustaining the ethical ideal of care in the twenty-first century. Philos Technol (2011) 24(3):251-68. doi:10.1007/ s13347-011-0015-x

24. Wada K, Shibata T, Musha T, Kimura S. Robot therapy for elders affected by dementia. Eng Med Biol Mag IEEE (2008) 27(4):53-60. doi:10.1109/ MEMB.2008.919496

25. Gould E, Maslow K, Lepore M, Bercaw L, Leopold J, Lyda-McDonald B, et al. Identifying and Meeting the Needs of Individuals with Dementia Who Live Alone Report. RTI Project Number 212050.035.001.001.001. (2015). Available from: https://www.researchgate.net/publication/303934162_Identifying_and_ Meeting_the_Needs_of_Individuals_With_Dementia_Who_Live_Alone

26. Johnston D, Samus QM, Morrison A, Leoutsakos JS, Hicks K, Handel S, et al. Identification of community-residing individuals with dementia and their unmet needs for care. Int J Geriatr Psychiatry (2011) 26:292-8. doi:10.1002/ gps. 2527

27. Miranda-Castillo C, Woods B, Orrell M. People with dementia living alone: what are their needs and what kind of support are they receiving? Int Psychogeriatr (2010) 22:607-17. doi:10.1017/S104161021000013X

28. Kobb R, Hilsen P, Ryan P. Assessing technology needs for the elderly: finding the perfect match for home. Home Healthc Nurse (2003) 21:666-73. doi:10.1097/00004045-200310000-00005

29. Likert R. A technique for the measurement of attitudes. Arch Psychol (1932) 140:55.

30. Zsiga K, Edelmayer G, Rumeau P, Péter O, Tóth A, Fazekas G. Home care robot for socially supporting the elderly: focus group studies in three European countries to screen user attitudes and requirements. Int J Rehabil Res (2013) 36(4):375-8. doi:10.1097/MRR.0b013e3283643d26

31. Douglas A, Letts L, Richardson J. A systematic review of accidental injury from fire, wandering and medication self-administration errors for older adults with and without dementia. Arch Gerontol Geriatr (2011) 52(1):e1-10. doi:10.1016/j.archger.2010.02.014

32. Rubenstein LZ, Josephson KR. Falls and their prevention in elderly people: what does the evidence show? Med Clin North Am (2006) 90:807-24. doi:10.1016/j.mcna.2006.05.013

33. Sterling DA, O'Connor JA, Bonadies J. Geriatric falls: injury severity is high and disproportionate to mechanism. J Trauma (2001) 50:116-9. doi:10.1097/00005373-200101000-00021

34. Bourgeois J, Couturier P, Tyrrell J. Safety at home for people with dementia: preliminary evaluation of situations-at-risk in a French geriatric memory clinic. Psychol Neuropsychiatr Vieil (2009) 7(3):213-24. doi:10.1684/pnv.2009.0174

35. National Center on Elder Abuse (NCEA), Administration on Aging (AOA). FAQs. (2015). Available from: https://ncea.acl.gov/faq/

36. Forlizzi J, DiSalvo C, Gemperle F. Assistive robotics and an ecology of elders living independently in their homes. Hum Comput Inter (2004) 19:25-59. doi:10.1207/s15327051hci1901\&2_3

37. Reddy R. Robotics and intelligent systems in support of society. IEEE Intell Syst (2006) 21:24-31. doi:10.1109/MIS.2006.57

38. Tanaka M, Ishii A, Yamano E, Ogikub H, Okazaki M, Kamimura K, et al. Effect of a human-type communication robot on cognitive function in elderly women living alone. Med Sci Monit (2012) 18(9):550-7. doi:10.12659/ MSM.883350

39. Kim GH, Jeon S, Im K, Kwon H, Lee BH, Kim GY, et al. Structural brain changes after traditional and robot-assisted multi-domain cognitive training in community-dwelling healthy elderly. 2015. PLoS One (2015) 10(4):e0123251. doi:10.1371/journal.pone.0123251

40. Rebok GW, Carlson MC, Langbaum JB. Training and maintaining memory abilities in healthy older adults: traditional and novel approaches. J Gerontol B Psychol Sci Soc Sci (2007) 62(1):53-61. doi:10.1093/geronb/62. special_issue_1.53

41. Williams K, Kemper S, Hummert ML. Enhancing communication with older adults: overcoming elderspeak. J Psychosoc Nurs Ment Health Serv (2005) 43:12-6. doi:10.3928/02793695-20050501-02 
42. Levin RP. Office communication is necessary for staff to maintain motivation. Dent Off (1989) 1(10):13.

43. Benítez Sandoval E, Brandstetter J, Obaid M, Bartneck C. Reciprocity in human-robot interaction: a quantitative approach through the prisoner's dilemma and the ultimatum game. Int J Soc Robot (2016) 8(2):303-17. doi:10.1007/s12369-015-0323-x

44. Frennert S, Eftring H, Östlund B. What older people expect of robots: a mixed methods approach. International Conference in Social Robots. Lecture Notes in Computer Science; 2013 Oct 27-29; Vol. 8239 Bristol, UK (2013). p. 19-29.

45. Carelli L, Gaggioli A, Pioggia G, De Rossi F, Riva G. Affective robot for elderly assistance. Stud Health Technol Inform (2009) 144:44-9. doi:10.3233/978-1-60750-017-9-44

46. Svanstrom R, Sundler AJ. Gradually losing one's foothold - a fragmented existence when living alone with dementia. Dementia (2015) 14(2):145-63. doi:10.1177/1471301213494510

47. Wang RH, Sudhama A, Begum M, Huq R. Robots to assist daily activities: views of older adults with Alzheimer's disease and their caregivers. Int Psychogeriatr (2016) 29(1):67-79. doi:10.1017/S1041610216001435

48. Begum M, Wang R, Huq R, Mihailidis A. Performance of daily activities by older adults with dementia: the role of an assistive robot. IEEE Int Conf Rehabil Robot (2013) 2013:6650405. doi:10.1109/ICORR.2013.6650405

49. Pino M, Boulay M, Jouen F, Rigaud AS. "Are we ready for robots that care for us"? Attitudes and opinions of older adults toward socially assistive robots. Front Aging Neurosci (2015) 7:141. doi:10.3389/fnagi.2015.00141

50. Heerink M, Kröse B, Evers V, Wielinga B. Assessing acceptance of assistive social agent technology by older adults: the Almere model. Int J Soc Robot (2010) 2:361-75. doi:10.1007/s12369-010-0068-5

51. Powers A, Kiesler S. The advisor robot: tracing people's mental model from a robot's physical attributes. Proceedings of the 1st ACM SIGCHI/SIGART Conference on Human-Robot Interaction. Salt Lake City (2006). p. 218-25.

52. Arras KO, Cerqui D. Do We Want to Share Our Lives and Bodies with Robots? A 2000 People Survey. Autonomous Systems Lab, Swiss Federal Institute of Technology Lausanne (EPFL) (2005). Technical Report Nr.0605-001.

53. Dautenhahn K. Methodology and themes of human-robot interaction: a growing research field. Int J Adv Robot Syst (2007) 4:103-8. doi:10.5772/5702

54. Rizvanoglu K, Ozturk O, Adiyaman O. The Impact of Human Likeness on the Older Adults' Perceptions and Preferences of Humanoid Robot Appearance, Design User Experience and Usability User Experience Design Practise, Volume 8520 of the Series Lecture Notes in Computer Science. Switzerland: Springer (2014). p. 164-72. e-ISSN 1611-3349, 20144939619.

55. Kulviwat S, Bruner GC, Kumar A, Nasco SA, Clark T. Toward a unified theory of consumer acceptance technology. Psychol Market (2007) 24:1059-84. doi:10.1002/mar.20196

56. Young JE, Hawkins R, Sharlin E, Igarashi T. Toward acceptable domestic robots: applying insights from social psychology. Int J Soc Robot (2009) 1(1):95-108. doi:10.1007/s12369-008-0006-y

57. Broadbent E, Stafford R, MacDonald B. Acceptance of healthcare robots for the older population: review and future directions. Int J Soc Robot (2009) 1:319. doi:10.1007/s12369-009-0030-6

58. Brandtstadter J, Renner G. Tenacious goal pursuit and flexible goal adjustment: explications and age-related analysis of assimilation and accommodation strategies of coping. Psychol Aging (1990) 5:58-67. doi:10.1037/ 0882-7974.5.1.58

59. Slangen-de Kort YAW, Midden CJH, van Wagenberg AF. Predictors of the adaptive problem-solving of older persons in their homes. J Environ Psychol (1998) 18(2):187-97. doi:10.1006/jevp.1998.0083
60. Bandura A. Self-efficacy: toward a unifying theory of behavioral change. Psychol Rev (1977) 84:191-215. doi:10.1037/0033-295X.84.2.191

61. Neven L. But obviously not for me: robots, laboratories and the defiant identity of elder test users. Sociol Health Illn (2010) 32:335-47. doi:10.1111/ j.1467-9566.2009.01218.x

62. Pain H, Gale CR, Watson C, Cox V, Cooper C, Sayer A. Readiness of elders to use assistive devices to maintain their independence in the home. Age Ageing (2007) 36:465-7. doi:10.1093/ageing/afm046

63. Mick DG, Fournier S. Paradoxes of technology: consumer cognizance, emotions, and coping strategies. J Consum Res (1998) 25:123-43. doi:10.1086/ 209531

64. Thielke S, Harniss M, Thompson H, Patel S, Demiris G, Johnson K. Maslow's hierarchy of human needs and the adoption of health-related technologies for older adults. Ageing Int (2012) 37:470-88. doi:10.1007/ s12126-011-9121-4

65. Zwijsen SA, Niemeijer AR, Hertogh CM. Ethics of using assistive technology in the care for community-dwelling elderly people: an overview of the literature. Aging Ment Health (2011) 15:419-27. doi:10.1080/13607863.2010.543662

66. Valkila N, Litja H, Aalto L, Saari A. Consumer panel study on elderly people's wishes concerning services. Arch Gerontol Geriatr (2010) 51(3):66-71. doi:10.1016/j.archger.2009.12.004

67. Dijkers MI, deBear PC, Erlandson RF, Kristy K, Geer DM, Nichols A. Patient and staff acceptance of robotic technology in occupational therapy: a pilot study. J Rehabil Res Dev (1991) 28:33-44. doi:10.1682/JRRD.1991.04.0033

68. Forlizzi J. Robotic products to assist the aging population. Interactions (2005) 12:16-8. doi:10.1145/1052438.1052454

69. Koay KL, Syrdal DS, Walters ML, Dautenhahn K. Living with robots: investigating the habituation effect in participants' preferences during a longitudinal human-robot interaction study. Proceedings of the 16th IEEE International Symposium on Robot and Human Interactive Communication RO-MAN. (Vol. 2007), Jeju Island, Korea (2007). p. 564-9.

70. Button KS, Ioannidis JP, Mokrysz C, Nosek BA, Flint J, Robinson ES, et al. Power failure: why small sample size undermines the reliability of neuroscience. Nat Rev Neurosci (2013) 14:365-76. doi:10.1038/nrn3475

71. Hair JF, Black WC, Babin BJ, Anderson RE, Tatham RL. Multivariate Data Analysis. (Vol. 6). Upper Saddle River, NJ: Pearson Prentice Hall (2006).

72. Charmaz K. Constructing Grounded Theory. Thousand Oaks, CA: SAGE (2014).

73. Johnson J, Gubrium JF, Holstein JA. In-Depth Interviewing. Handbook of Interview Research: Context and Method. Thousand Oaks, CA: SAGE (2002). p. 103-19.

74. Morgan D. Focus Groups as Qualitative Research. Newbury Park, CA: SAGE (1988).

Conflict of Interest Statement: The authors declare that the research was conducted in the absence of any commercial or financial relationships that could be construed as a potential conflict of interest.

Copyright $\odot 2017$ Korchut, Szklener, Abdelnour, Tantinya, Hernández-Farigola, Ribes, Skrobas, Grabowska-Aleksandrowicz, Szcześniak-Stańczyk and Rejdak. This is an open-access article distributed under the terms of the Creative Commons Attribution License (CC BY). The use, distribution or reproduction in other forums is permitted, provided the original author(s) or licensor are credited and that the original publication in this journal is cited, in accordance with accepted academic practice. No use, distribution or reproduction is permitted which does not comply with these terms. 